\title{
The global dust modelling framework THEMIS
}

\author{
A. P. Jones ${ }^{1}$, M. Köhler ${ }^{2}$, N. Ysard ${ }^{1}$, M. Bocchio ${ }^{1}$, and L. Verstraete ${ }^{1}$ \\ 1 Institut d'Astrophysique Spatiale, CNRS, Université Paris-Sud, Université Paris-Saclay, Bât. 121, 91405 Orsay Cedex, France \\ e-mail: Anthony. Jones@ias.u-psud.fr \\ 2 School of Physics and Astronomy, Queen Mary, University of London, 327 Mile End Road, London, E1 4NS, UK
}

Received 9 December 2016 / Accepted 1 March 2017

\begin{abstract}
Here we introduce the interstellar dust modelling framework THEMIS (The Heterogeneous dust Evolution Model for Interstellar Solids), which takes a global view of dust and its evolution in response to the local conditions in interstellar media. This approach is built upon a core model that was developed to explain the dust extinction and emission in the diffuse interstellar medium. The model was then further developed to self-consistently include the effects of dust evolution in the transition to denser regions. The THEMIS approach is under continuous development and we are currently extending the framework to explore the implications of dust evolution in HII regions and the photon-dominated regions associated with star formation. We provide links to the THEMIS, DustEM and DustPedia websites where more information about the model, its input data and applications can be found.
\end{abstract}

Key words. dust, extinction - ISM: general

\section{Introduction}

Interstellar dust has been a major topic in observational studies for more than 80 years, beginning with the early studies of interstellar absorption. Trumpler (1930) studied interstellar reddening or selective absorption towards open star clusters but the effect had also, and independently, been discovered by Schalén (1929, 1931). However, Schalén's work on this subject in the early 1930s has largely been forgotten (Hockey et al. 2014). Dust modelling has had a long history, with the first attempts at dust modelling appearing soon after Trumpler and Schalén's interstellar absorption studies. Indeed, Schalén (1934) and Schoenberg \& Jung (1934) used Gustave Mie's theory (Mie 1908) to study the diffusion of starlight by interstellar matter and found that the interstellar absorption could be reproduced by metallic particles. Sometime later followed the so-called "dirty ice" model proposed by van de Hulst (1943), which was further developed by Oort \& van de Hulst (1946). In the latter work Oort and van de Hulst determined that accretion in the interstellar medium (ISM) on a time-scale $\simeq 10^{8} \mathrm{yr}$ would form grains with radii of $100 \mathrm{~nm}$. In their scenario accretion is balanced by dust destruction in cloud-cloud collisions once every $\simeq 10^{8} \mathrm{yr}$, leading to a grain life-time of $\simeq 50$ million years. Thus, dust evolution in the ISM has been an important consideration since the first dust models were developed.

It was almost two decades after the dirty ice model was proposed that small graphite flakes, assumed to be formed around cool carbon stars, were proposed as an alternative dust material to explain the visible to near-IR extinction (Hoyle \& Wickramasinghe 1962). Soon after, silicate and graphite grains, with and without ice mantles, were shown to be consistent with the observed extinction (Wickramasinghe 1963, 1970). With the measurement of the UV extinction and the discovery of the UV bump at $\sim 217 \mathrm{~nm}$ (Stecher 1965, 1969) graphite became a preferred dust material and a likely UV bump-carrier (Stecher \& Donn 1965). At about the same time, it was proposed that graphite along with silicate grains, formed around oxygen-rich giant stars, are the major interstellar dust species (Hoyle \& Wickramasinghe 1969). So was born the graphite and silicate dust model, based on laboratory-measured optical constants, which after almost fifty years remains the basis of almost all current dust models. In the late seventies, using laboratory-measured optical constants, Mathis et al. (1977) explored the viability of un-coated graphite, enstatite, olivine $\mathrm{SiC}$, iron and magnetite as suitable interstellar dust analogues. From this they concluded that a viable model for interstellar extinction must include graphite and that it, in combination with any of the other materials, could give a good fit to the observed extinction, provided that power law grain size distributions were assumed (Mathis et al. 1977, hereafter MRN). The preferred MRN dust model was for graphite grains partnered with an olivine-type silicate. Basing their work upon the MRN dust model Draine \& Lee (1984) semi-empirically tuned graphite and silicate optical properties to fit observations, resulting in "astronomical" graphite and silicate. Polycyclic aromatic hydrocarbon (PAH) molecules were later added into the mix (Desert et al. 1990; Siebenmorgen \& Kruegel 1992; Dwek et al. 1997; Draine \& Li 2001, 2007; Li \& Draine 2001, 2002; Siebenmorgen et al. 2014) but the physical basis for these models, even including the later improvements, is fundamentally little different from the MRN model. It should be noted that in all of these models the different grain populations, for example, graphite and olivine (MRN), astronomical graphite and silicate (Draine \& Lee 1984) or astronomical graphite, silicate and PAH (Siebenmorgen \& Kruegel 1992; Dwek et al. 1997; Draine \& Li 2001, 2007; Li \& Draine 2001, 2002; Siebenmorgen et al. 2014) are considered to reside in distinct and separate dust populations. Some later dust models followed this same basic approach but abandoned astronomical graphite in favour of physically more-realistic amorphous carbons (e.g., Zubko et al. 2004; Compiègne et al. 2011; Galliano et al. 2011). However, most of these models also suffer the same limitations as all MRN-based 
models, that is "The particles responsible for the $2200 \AA$ hump and the FUV extinction constitute two independent populations, and the MRN graphite plus silicate model violates this condition". (Greenberg \& Chlewicki 1983) and "The ultraviolet extinction by the particles responsible for the visual extinction is grey and therefore these particles constitute an entirely separate population from the hump particles or the FUV particles". (Greenberg \& Chlewicki 1983). In contrast, the Desert et al. (1990) dust model was built to be consistent with Greenberg \& Chlewicki (1983) requirements and was something of a departure from previous models in that it introduced PAHs, assumed that the small grains are made of an amorphous carbon material and adopted big silicate grains with a "dark refractory mantle" of hydrocarbon composition.

Given the turbulent nature of the ISM and the observation that the depletions of the dust-forming elements vary significantly and differentially (e.g., Routly \& Spitzer 1952; Crinklaw et al. 1994; Savage \& Sembach 1996; Jones 2000; Welty et al. 2002; Jenkins 2009; Parvathi et al. 2012) it is difficult to see how the different dust materials could remain distinct. The cycling of gas and dust through different ISM phases naturally requires that the dust materials become mixed into inhomogeneous assemblages. This mixing could be in the form of graphite and silicate core icemantled grains (Wickramasinghe 1963) or refractory-mantled particles, along the lines of those originally proposed by Greenberg (1986), in porous particles or mixed-material aggregates (e.g., Jones 1988; Mathis \& Whiffen 1989). Indeed, physically-viable interstellar dust models based on silicate grains with "organic" or (hydrogenated) amorphous carbon mantles have been around for more than $30 \mathrm{yr}$ (e.g., Greenberg 1986 Duley 1987; Jones et al. 1987, 1990, 2013, 2014; Duley et al. 1989; Jones 1990; Sorrell 1991; Li \& Greenberg 1997; Iatì et al. 2008; Cecchi-Pestellini et al. 2010; Zonca et al. 2011; Cecchi-Pestellini et al. 2014a,b; Köhler et al. 2014). As per all dust models, variations in the grain size distribution can be used to explain the observed variations in dust emission and extinction. However, a major advantage of the core-mantle models is that they can, in addition and quite naturally, account for dust variations through environmentally-driven changes in the carbonaceous mantle composition and/or its depth (e.g., Jones et al. 1987, 1990, 2013, 2016; Köhler et al. 2014; Duley et al. 1989; Li \& Greenberg 1997; Cecchi-Pestellini et al. 2010; Zonca et al. 2011; Cecchi-Pestellini et al. 2014a,b; Ysard et al. 2015, 2016).

The long-standing and widely-adopted idea of core-mantle (CM) interstellar grains was recently given a new treatment (Jones et al. 2013; Köhler et al. 2014). The result of this is the THEMIS dust modelling framework, which provides a coremantle model for dust in the diffuse ISM and the evolution of the dust properties in response to their local environment (Jones et al. 2013, 2014, 2016; Köhler et al. 2014; Bocchio et al. 2014; Ysard et al. 2015, 2016). The underlying principle of the THEMIS modelling framework is the supposition that interstellar dust is not the same everywhere but that it evolves within a given region of the ISM as it reacts to and interacts with its local environment. Indeed, variations in the dust properties from one region to another have long been interpreted as due to dust evolution (e.g., Lefevre 1974; Jura 1980; Aannestad \& Greenberg 1983; Whittet et al. 1992, 2001; Ossenkopf 1993; Stepnik et al. 2003). For example, photon, ion and electron irradiation can induce changes in the dust chemical composition and structure (e.g., Demyk et al. 2001, 2004), hydrogenation and accretion can drive changes in the grain chemical composition (e.g., Hecht 1986; Sorrell 1990, 1991; Mennella 2008, 2010; Jones 2012a) and accretion/coagulation will change the grain structure (e.g., Köhler et al. 2011, 2012, 2015; Jones et al. 2014, 2016). All of these processes directly affect the dust optical properties (i.e., the particle absorption and scattering cross-sections) as the composition, structure and shape of the grains evolve as they transit from one region to another. For example, in the tenuous ISM the outer carbonaceous layers of the grains, be they carbon grains or the mantles on other grains, will be H-poor and aromatic rich due to UV photolysis by stellar FUV/EUV photons (e.g., Hecht 1986; Sorrell 1990, 1991; Jones 2012a,b,c, 2016a; Jones et al. 2013) but in denser regions accreted hydrocarbon mantles are likely H-rich and aromatic poor (Jones et al. 2014, 2016; Ysard et al. 2016).

In our dust modelling studies we consider the dust properties as they evolve in response to the physical conditions in their local environment, e.g., density, radiation field (intensity and hardness as effected by extinction) and kinematics/dynamics. This evolution drives the dust properties (structure and composition) towards a state that is assumed to be in equilibrium with the given local conditions. For example, in diffuse and dense clouds, where the dust seemingly resides undisturbed for millions of years, the typical dust processing time-scales are short with respect to the dynamical time-scales and the dust is generally in equilibrium with the local conditions in these regions. However, in photon-dominated regions (PDRs) and shocked regions this is no longer the case because the dynamical time-scales are significantly shorter and the dust is often out of equilibrium and constantly evolving in response to the local physical conditions.

Here and from now on we will refer to our evolutionary dust modelling approach, and all future developments and extensions of it, under the umbrella acronym THEMIS (The Heterogeneous dust Evolution Model for Interstellar Solids) ${ }^{1}$.

\section{The THEMIS dust modelling framework}

The central tenet of THEMIS is that interstellar dust is not the same everywhere but that it reacts to its local environment. The core dust model central to the THEMIS approach (see Sect. 2.2) is built, as much as has been possible, upon the foundations of the laboratory-measured properties of physicallyreasonable interstellar dust analogue materials, i.e., amorphous olivine-type and pyroxene-type silicates with iron and iron sulphide nano-inclusions, a-Sil $\mathrm{Fe}_{\mathrm{FeS}}$, and the extensive family of hydrogenated amorphous carbon materials, a-C(:H) (Jones et al. 2013; Köhler et al. 2014; Jones 2015). In constructing and developing the THEMIS framework we consider that the physical properties of a-Sil $\mathrm{Fe}_{\text {,FeS }}$ and, in particular, of a-C $(: \mathrm{H})$ materials and their structural juxtapositions evolve in a coherent and selfconsistent manner within a given region of the ISM. For example, irradiation can induce changes in the dust chemical composition and structure, and changes in structure can also be driven by accretion and coagulation. All of these processes directly affect the dust optical properties (absorption and scattering crosssections), which are the key to understanding the nature of dust. In our modelling we take into account the shape of the particles and the evolution of the shape distribution along a sequence of increasingly complex particle shapes (see Sect. 2.3). Shape effects are particularly important in the case of coagulated particles and grains with incomplete and/or non-uniform mantles (e.g., Köhler et al. 2012). For complex grain shapes we calculate the optical properties using DDSCAT (Draine \& Flatau 2000), as

\footnotetext{
In previous publications THEMIS was the acronym for The Heterogeneous dust Evolution Model at the Institut d'Astrophysique Spatiale.
} 
described elsewhere (Köhler et al. 2011, 2012, 2015). We have not yet considered the polarising properties of such complex grain structures.

In the following sub-sections we highlight the most important aspects of THEMIS and how this framework approach will be developed in the future.

\subsection{Dust structural and optical properties}

At the core of any model for interstellar dust are the input optical constants, the complex indices of refraction $(m=n+i k)$, which are used to derive the dust cross-sections (optical properties), for extinction $(i=\mathrm{ext})$, absorption $(i=\mathrm{abs})$ and scattering $(i=\mathrm{sca})$,

$C_{i}(a, \phi, \xi, \lambda)=\pi a^{2} \times Q_{i}(a, \phi, \xi, \lambda)$,

which are a function of the particle radius $(a)$, material $(\phi)$, grain structure $(\xi)$ and the wavelength $(\lambda)$. We have as much as possible based our dust model on the best available, laboratory-measured or laboratory-derived properties of analogues of interstellar dust materials: amorphous silicates (Scott \& Duley 1996), hydrogenated amorphous carbons (Smith 1984; Duley 1984; Rouleau \& Martin 1991; Mennella et al. 1995; Zubko et al. 1996), iron (Ordal et al. 1983, 1985, 1988), iron sulphide (Pollack et al. 1994) and water ice (Warren 1984). Where the available data do not cover the required (wavelength) range we have extrapolated those data in as physicallymeaningful way as possible. Further, where suitable data were not available we have constructed new data "ground-up" using fundamental principles and have calibrated these data on the available laboratory measurements (Jones 2012a,b,c; Köhler et al. 2014).

\subsubsection{Amorphous silicates, a-Sil}

In the case of amorphous silicates, a-Sil, there is a wealth of laboratory data and it can be difficult to determine which is the most appropriate analogue material data to use to model interstellar silicates. Hence, in our choice, we were motivated by experimental data, astronomical observations, direct interstellar dust analyses and interstellar depletion studies of, principally, $\mathrm{Mg}, \mathrm{Si}, \mathrm{Fe}$ and S. Our final choice of mixed amorphous silicates with metallic iron and iron sulphide nano-inclusions was motivated by three major lines of evidence. A fourth piece of evidence subsequently came to light and thus adds weight to our earlier assumptions.

Firstly, observations of silicate dust in the ISM towards the Galactic Centre indicate that they are a mix of amorphous olivine-type and pyroxene-type silicates (e.g., Chiar et al. 2000).

Secondly, the work of Davoisne et al. (2006) on the annealing of iron-bearing amorphous silicate shows that in the presence of carbon the iron is reduced to metal nano-inclusions within an Mg-rich amorphous silicate matrix. This structure is much akin to the Glass with Embedded Metal and Sulphide (GEMS) component of interplanetary dust particles (e.g., Bradley 1994).

Thirdly, X-ray observational data indicate that even though iron is present in interstellar dust it is not in Fe-rich silicates but in the form of metallic iron inclusions within $\mathrm{Mg}$-rich silicates (e.g., Costantini et al. 2005, 2012, Xiang et al. 2011)

Fourthly, and subsequent to the publication of the original Jones et al. (2013) diffuse ISM dust model, seven particles of interstellar origin collected and analysed by the Stardust mission exhibit amorphous and crystalline grains and multiple iron-bearing phases, including metallic iron and iron sulphide (Westphal et al. 2014).
For interstellar amorphous silicate dust, a-Sil, we use a 1:1 by mass mix of amorphous forsterite-type and enstatite-type silicates (with the complex refractive indices $n$ and $k$ taken from Scott \& Duley 1996) with iron incorporated in the form of metallic iron iron ( $n$ and $k$ from Ordal et al. 1983, 1985, 1988) and iron sulphide ( $n$ and $k$ from Pollack et al. 1994) nano-particle inclusions occupying $7 \%$ and $3 \%$ of the grain volume, respectively (a-Sil $_{\mathrm{Fe}, \mathrm{FeS}}$, Jones et al. 2013; Köhler et al. 2014) ${ }^{2}$.

\subsubsection{Amorphous silicate mass densities}

In our dust modelling we assume an amorphous silicate material density of $2.5 \mathrm{~g} \mathrm{~cm}^{-3}$, which has probably never before been justified. The assumption of a lower density, than for bulk silicate materials, is adopted because, firstly, amorphous materials are less dense than their crystalline cousins and, secondly, sub- $\mu \mathrm{m}$ particles will not have the same density as their larger siblings or their parental solids because of surface effects.

For the crystalline silicate mineral equivalents of the amorphous silicate materials that we use, i.e., enstatite-type pyroxene $\left(\mathrm{MgSiO}_{3}\right)$ and forsterite-type olivine $\left(\mathrm{Mg}_{2} \mathrm{SiO}_{4}\right)^{3}$, the bulk material densities are $3.2 \mathrm{~g} \mathrm{~cm}^{-3}$ and $3.3 \mathrm{~g} \mathrm{~cm}^{-3}$, respectively. The assumed a-Sil density is therefore $\simeq 20 \%$ lower than the equivalent crystalline silicate material density.

While is it hard to find direct measurements of the densities of enstatite-type and forsterite-type amorphous silicate nanoparticles, it has been shown that silica $\left(\mathrm{SiO}_{2}\right)$ nano-particles $\left(a \simeq 30-200 \mathrm{~nm}\right.$ ) have densities of $\sim 1.9 \mathrm{~g} \mathrm{~cm}^{-3}$ (Kimoto et al. 2014) compared to a bulk material density of $2.2-2.7 \mathrm{~g} \mathrm{~cm}^{-3}$. The density of silica nano-particles is therefore reduced by $\simeq 14-30 \%$ with respect to that of the parental solid. Thus, and by analogy with silica, a density reduction of $\simeq 20 \%$ for sub- $\mu \mathrm{m}$ amorphous silicate particles would appear to be supported by laboratory measurements (Kimoto et al. 2014).

\subsubsection{Hydrogenated amorphous carbons, $a-C(: H)$}

Finding definitive laboratory data sets for physically-reasonable analogues of interstellar carbonaceous materials is difficult because of the wide range in properties of these materials (e.g., Robertson 1986, 1988, Robertson \& O'Reilly 1987). Graphite has traditionally been used in many dust models but, while there are rather rare pre-solar graphite grains in primitive meteorites, there appears to be no evidence to support it as the most abundant interstellar carbon dust material (e.g., Dartois et al. 2004b, 2005; Serra Díaz-Cano \& Jones 2008; Compiègne et al. 2011). However, amorphous hydrocarbons do appear to be an important component of the dust in the Milky Way (e.g., Pendleton \& Allamandola 2002) and also in galaxies (e.g., Dartois et al. 2004a). These materials are consistent with the observed infrared absorption bands (e.g., Pendleton \& Allamandola 2002; Dartois et al. 2004b; Dartois \& Muñoz-Caro 2007) and show temperaturedependent luminescence (e.g., Robertson 1986) consistent with the observed interstellar luminescence in the red, the so-called extended red emission (ERE, Duley et al. 1997; Duley 2001; Dartois et al. 2005; Godard \& Dartois 2010)

\footnotetext{
2 The complex refractive indices were calculated separately for each a-Sil material mixed with $\mathrm{Fe}, \mathrm{FeS}$ nano-inclusions using the Garnett effective medium theory (EMT, Garnett 1904).

3 N.B. The amorphous equivalents of the crystalline silicate should be referred to as of -type because these specific mineral names only refer to crystalline materials.
} 
Table 1. THEMIS model elemental abundance requirements (in ppm).

\begin{tabular}{lccccccccccc}
\hline \hline $\begin{array}{l}\text { Dust } \\
\text { component }\end{array}$ & $\begin{array}{c}\mathrm{C} \\
(\mathrm{a}-\mathrm{C}: \mathrm{H})\end{array}$ & $\begin{array}{c}\mathrm{C} \\
(\mathrm{a}-\mathrm{C})\end{array}$ & $\begin{array}{c}\mathrm{C} \\
\text { (total) }\end{array}$ & $\mathrm{O}$ & $\mathrm{Mg}$ & $\mathrm{Si}$ & $\begin{array}{c}\mathrm{Fe} \\
(\mathrm{metal})\end{array}$ & $\begin{array}{c}\mathrm{Fe} \\
(\mathrm{FeS})\end{array}$ & $\begin{array}{c}\text { density } \\
\left(\mathrm{g} / \mathrm{cm}^{3}\right)\end{array}$ & $M_{\mathrm{d}} / M_{\mathrm{H}}$ \\
\hline Nano a-C & $13-4$ & $130-140$ & $143-144$ & - & - & - & - & - & - & 1.60 & $1.7 \times 10^{-3}$ \\
Large a-C:H/a-C & $45-39$ & $5-13$ & $50-52$ & - & - & - & - & - & - & $1.51-1.57$ & $(6.0-6.3) \times 10^{-4}$ \\
Large a-Sil/a-C & - & $13-22$ & $13-22$ & 110 & 45 & 32 & 16 & 3 & 3 & $2.19-1.94$ & $(5.1-4.5) \times 10^{-3}$ \\
\hline
\end{tabular}

Notes. Variations in the diffuse ISM dust emission and extinction (Ysard et al. 2015) can be explained with a range of a-C mantle depths (thin-thick, see text), which do not significantly affect the total carbon abundance.

and with the likely irradiation effects and the associated aliphatic to aromatic transformation in the ISM (e.g., Jones 1990; Dartois et al. 2004a,b; Pino et al. 2008; Mennella 2008; Godard et al. 2011), in circumstellar regions (e.g., Goto et al. 2003, 2007; Sloan et al. 2007; Pino et al. 2008), in interplanetary dust particles (IDPs, Muñoz Caro et al. 2006) and solar system organics (Dalle Ore et al. 2011). Thus, the most physically-realistic carbonaceous grain materials, albeit inherently rather complex, would appear to be the extensive family of (hydrogenated) amorphous carbons, a-C $(: \mathrm{H})^{4}$. Hydrogenated amorphous carbon materials, a-C $(: \mathrm{H})$, are macroscopically-structured, contiguous network, solid-state materials comprised of only carbon and hydrogen atoms. The properties of a-C(:H) materials have been well-studied within both the physics and astrophysics communities (e.g., Phillips 1979; Döhler et al. 1980; Thorpe 1983; Robertson 1986, 1988, 1991, 2001, 2002; Robertson \& O'Reilly 1987; Angus \& Jansen 1988; Angus \& Hayman 1988; Tamor \& Wu 1990; Mennella et al. 1995, 1996, 2003; Ferrari \& Robertson 2000, 2004; Kassavetis et al. 2007; Llamas-Jansa et al. 2007; Mennella 2008; Gadallah et al. 2011; Jones 2012a,b,c).

There is a large database of laboratory-measured (e.g., Smith 1984; Duley 1984; Mennella et al. 1995; Gavilan et al. 2016) ${ }^{5}$ and post-processed (e.g., Rouleau \& Martin 1991; Zubko et al. $1996)^{6}$ optical property data for a-C(:H) materials but it is far from homogeneous in terms of composition/structure, synthesis methods and wavelength coverage. Hence, until such time as the gaps have been filled we rely upon a recentlydeveloped set of optical properties for a large part of the a-C $(: \mathrm{H})$ family parameter space (Jones 2012a,b,c). These optical properties were built ground-up using random covalent network (RCN) models (Phillips 1979; Döhler et al. 1980; Thorpe 1983; Angus \& Jansen 1988) and extensions thereof (extended RCN (eRCN) and Defective Graphite (DG) models, Tamor \& Wu 1990; Jones 1990, 2012a) to construct a solid-state framework method to derive their structure and thence their complex indices of refraction. This approach determines the compositionand size-dependent optical properties of a-C $(: \mathrm{H})$ materials (the $\operatorname{optEC}_{(\mathrm{s})}$ and $\operatorname{optEC}_{(\mathrm{s})}(\mathrm{a})$ datasets, Jones 2012a,b,c) based upon eRCN and DG modelling methods (Jones 1990, 2012a). Using this approach the likely evolution of hydrocarbon solids in the ISM was elucidated and explored in detail, even down to

\footnotetext{
4 These semiconducting materials span a wide compositional range; from $\mathrm{H}$-poor, aromatic-rich a-C with $\lesssim 15$ at. $\% \mathrm{H}$ and narrow band gap $\left(E_{\mathrm{g}}<1 \mathrm{eV}\right)$ to $\mathrm{H}-$ rich, aliphatic-rich a-C:H with $\sim 15-60$ at. $\% \mathrm{H}$ and wide band gap $\left(E_{\mathrm{g}} \geq 1 \mathrm{eV}\right)$.

5 See also the Jena group's "Databases of Dust Optical Properties" http://www.astro.uni-jena.de/Laboratory/Database/ databases.html

6 N.B. These two post-processed data sets, derived from similar laboratory data, arrive at divergent solutions for the optical properties of their amorphous carbons at far- to extreme-UV wavelengths.
}

molecular dimensions, by Jones (2012a,b,c). THEMIS builds upon this foundation, which was used to construct a diffuse ISM dust model (Jones et al. 2013; Köhler et al. 2014), using the $\operatorname{optEC}_{(\mathrm{s})}(a)$ optical property data ${ }^{7}$ to predict and explore the observed interstellar dust extinction and emission properties (see following section).

\subsection{The diffuse ISM dust model}

THEMIS makes the fundamental assumption that the silicate and carbonaceous dust populations cannot be completely segregated in the ISM and that amorphous silicate grains are mixed with a carbonaceous dust component (e.g., Mathis \& Whiffen 1989; Desert et al. 1990), most likely in core/mantle (CM) structures (e.g., Greenberg 1986; Duley 1987; Jones et al. 1987, 1990; Duley et al. 1989; Li \& Greenberg 1997). The core of the THEMIS approach is its diffuse ISM dust model (Jones et al. 2013; Köhler et al. 2014; Ysard et al. 2015), which comprises the following grain and core/mantle ${ }^{8}(\mathrm{CM})$ grain structures and compositions:

- a power-law distribution of a-C nano-particles ( $a \lesssim 20 \mathrm{~nm}$ ) with strongly size-dependent optical properties;

- a log-normal distribution $\left(a \simeq 10-3000 \mathrm{~nm}, a_{\text {peak }} \simeq 160 \mathrm{~nm}\right)$ of large a-C(:H) CM grains with UV photolysed a-C surface layers (mantle depth $=7.5-20 \mathrm{~nm}$ ) surrounding a-C:H cores; and

- a log-normal distribution $\left(a \simeq 10-3000 \mathrm{~nm}, a_{\text {peak }} \simeq 140 \mathrm{~nm}\right)$ of large a-Sil $l_{\mathrm{Fe}, \mathrm{FeS}}$ grains with a-C mantles (depth $\left.=5-10 \mathrm{~nm}\right)$ formed by carbon accretion and/or by the coagulation of small a-C particles onto their surfaces.

This diffuse ISM dust model uses $\simeq 206-218 \mathrm{ppm}$ of carbon (with $\simeq 143-144 \mathrm{ppm}$ in the a-C nano-particles), $110 \mathrm{ppm}$ of oxygen, $45 \mathrm{ppm}$ of magnesium, $32 \mathrm{ppm}$ of silicon, $19 \mathrm{ppm}$ of iron and $3 \mathrm{ppm}$ of sulphur. The Fe and $\mathrm{S}$ are incorporated within the amorphous silicate grains in the form of $\mathrm{Fe}$ and FeS nano-inclusions, with $7 \%$ and $3 \%$ volume filling fractions, respectively (Jones et al. 2013; Köhler et al. 2014; Ysard et al. 2015). The breakdown of these abundances, along with the size-dependent grain material densities and the required dust masses for the above diffuse ISM model are detailed in Table 1. The resulting nano-inclusion-containing a-Sil effective densities, $\left\langle\rho_{\mathrm{a}-\mathrm{Sil}}\right\rangle$, are given by:

$\left\langle\rho_{\mathrm{a}-\mathrm{Sil}}\right\rangle=\rho_{\mathrm{a}-\mathrm{Sil}}\left(1-f_{\mathrm{Fe}}-f_{\mathrm{FeS}}\right)+f_{\mathrm{Fe}} \rho_{\mathrm{Fe}}+f_{\mathrm{FeS}} \rho_{\mathrm{FeS}}$,

where $\rho_{\mathrm{a}-\mathrm{Sil}}, \rho_{\mathrm{Fe}}$ and $\rho_{\mathrm{FeS}}$ are the assumed material densities $\left(2.5,7.87\right.$ and $4.84 \mathrm{~g} \mathrm{~cm}^{-3}$, respectively), and $f_{\mathrm{Fe}}(0.07)$ and

\footnotetext{
7 Available at: http://cdsarc.u-strasbg.fr/viz-bin/qcat?]/ $\mathrm{A}+\mathrm{A} / 545 / \mathrm{C} 2$ and http: //cdsarc.u-strasbg.fr/viz-bin/qcat? $\mathrm{J} / \mathrm{A}+\mathrm{A} / 545 / \mathrm{C} 3$

8 In this context a slash is used here and hereafter to indicate a separation between two compositionally-distinct layers (see Fig. 1).
} 
$f_{\mathrm{FeS}}(0.03)$ are the volume filling fractions of $\mathrm{Fe}$ and FeS. The effective density of the a-C coated amorphous silicate (with nanoinclusions), and also of the large a-C:H/a-C CM grains, is then given by:

$$
\begin{aligned}
\left\langle\rho_{\mathrm{CM}}\right\rangle & =\frac{m_{\text {core }}+m_{\text {mantle }}}{\frac{4}{3} \pi a^{3}} \\
& =\frac{\frac{4}{3} \pi\left\langle\rho_{\text {core }}\right\rangle(a-d)^{3}+\frac{4}{3} \pi \rho_{\text {mantle }}\left[a^{3}-(a-d)^{3}\right]}{\frac{4}{3} \pi a^{3}} \\
& =\left\langle\rho_{\text {core }}\right\rangle\left[1-(d / a)^{3}\right]+\rho_{\text {mantle }}\left\{1-\left[1-(d / a)^{3}\right]\right\},
\end{aligned}
$$

where $a$ is the outer grain radius, $d$ is the mantle depth, $m_{\text {core }}$ and $m_{\text {mantle }}$ are the grain mantle and core masses and $\rho_{\text {core }}$ and $\rho_{\text {mantle }}$ are their material densities. Given that in the THEMIS model the mantle thicknesses are independent of particle radius, the determination of grain densities, and consequently of the elemental abundances, requires integration over the entire size distribution for each dust component.

We note that if the amorphous silicate $\mathrm{Fe}$ and $\mathrm{FeS}$ nanoinclusion densities are also reduced by $\simeq 20 \%$, as for amorphous silicate materials, something that we have not included in our model, then the total $\mathrm{Fe}$ and $\mathrm{S}$ abundance requirements given in Table 1 would be reduced by less than $1 \mathrm{ppm}$.

\subsection{Dust evolution within dense regions}

The diffuse ISM dust model (Sect. 2.2) was extended in order to explore the evolution of the dust optical properties in the transition to denser molecular cloud regions in as self-consistent a way as possible (Köhler et al. 2012, 2015). In THEMIS this evolution is assumed to result in the following core/mantle/mantle $(\mathrm{CMM})$ grains and their aggregated forms (AMM) with ice mantles $(\mathrm{AMMI})^{9}$ :

- CMM: a-C-mantled a-Sil $\mathrm{Fe}, \mathrm{FeS}$ and a-C:H/a-C CM grains with an additional outer mantle of a-C:H formed by the accretion of remnant gas phase carbon with a composition that is determined by the local conditions (radiation field and gas density);

- AMM: Aggregates of CMM grains;

- AMMI: Aggregates of CMM grains with ice mantles.

The accretion/coagulation-evolved dust compositions and structures, with size distributions similar to log-normal (Köhler et al. 2015), are entirely consistent with the observed evolution of the dust properties in the transition towards denser regions (Köhler et al. 2012; Jones et al. 2014), including a decrease in the dust temperature and an increase in the dust spectral index and opacity at far-IR/sub-mm wavelengths (Köhler et al. 2015). The accretion of a-C:H mantles in denser regions is also consistent with both cloud- and core-shine (C-shine) observations (Jones et al. 2016; Ysard et al. 2016). Figure 1 shows a schematic view of the above-described dust evolution stages and the associated grain structures and compositions. The relevant time-scales for accretion and coagulation, as a function of depth into a molecular cloud, were presented in our previous work (Köhler et al. 2012; Jones et al. 2014) and are therefore not repeated here.

For the diffuse cloud model the dust optical properties were calculated using the Mie theory for coated spheres (e.g., Bohren \& Huffman 1998) and for more complex structures, the multi-component (CMM) and aggregate grain

\footnotetext{
9 We take the optical constants of ice from Warren (1984).
}

(AMM and AMMI) optical properties were calculated using the discrete-dipole approximation (DDA) method (DDSCAT, Draine \& Flatau 2000).

The evolutionary changes in the dust structure, composition and dust size distribution, outlined above, are self-consistent and, principally due to the transformation and loss of the smallest grains through mantle accretion and coagulation onto larger grains, respectively. A full time-dependent and self-consistent study of dust accretion and coagulation in the diffuse to dense cloud transition is not yet possible. This is because the calculation of the grain optical properties, which is currently best performed with DDA, is time-consuming for complex grain structures at short wavelengths. However, these calculations now only need to be made for new grain structures. Thus, and for now, we assume a "stepped" approach to dust evolution, i.e., we work with a limited number of size distributions and compositions that approximate the continuity of the evolutionary processes operating in dense regions.

\subsection{Dust evolution in PDRs and HIl regions}

In our forthcoming work we will explore the nature and the detailed physical evolution of the dust in PDRs. In these regions the dust evolution is complex and likely cannot be modelled as a simple reversal of the construction processes that lead to the assembly of complex aggregates in molecular clouds. This lack of symmetry is due to the more intense radiation fields in PDRs compared to molecular clouds, which introduces a strong hysteresis effect.

The THEMIS approach has led to suggestions for viable routes for the formation of molecular hydrogen and of daughter hydrocarbon species in moderately excited PDRs (Jones 2012b; Jones \& Habart 2015). The proposed photolytic route, which has been measured in the laboratory (e.g., Smith 1984; Adel et al. 1989; Marée et al. 1996; Godard et al. 2011; Alata et al. 2014), could act in conjunction with and complement other formation mechanisms (e.g., Hollenbach \& Salpeter 1971; Rauls \& Hornekær 2008; Le Page et al. 2009; Bron et al. 2014). We have continued this work with an investigation of the effects of grain charging and the complimentary effects of gas heating, via photo-electron emission, and gas cooling (Bocchio et al., in prep.). The THEMIS model appears to be consistent with the latest observations of gas heating in the diffuse ISM (Bocchio et al., in prep.). We are currently also exploring the likely response of the THEMIS model a-C $(: \mathrm{H})$ nano-particles to hard UV photo-processing in PDRs and HII regions, with a view to understanding nano-particle evolution in excited regions.

\subsection{Dust processing in high energy environments}

A model of the critical processing of small particles in electron and ion interactions in shocks and cosmic rays interactions was developed by Micelotta et al. (2010b,a, 2011). We extended this methodology to the small a-C particles in THEMIS (Bocchio et al. 2012) and used it to study the effects of electron collisional heating and dust erosion in a hot coronal gas (Bocchio et al. 2013). We have also studied the effects of dust processing and destruction in supernova-driven shock waves using the THEMIS diffuse ISM model (Bocchio et al. 2014).

In these environments the smallest grains appear to have rather short lifetimes and cannot be collisionally heated without also being (partly) destroyed. Recent work by Wolf et al. (2016) shows that the so-called super-hydrogenated polycyclic 


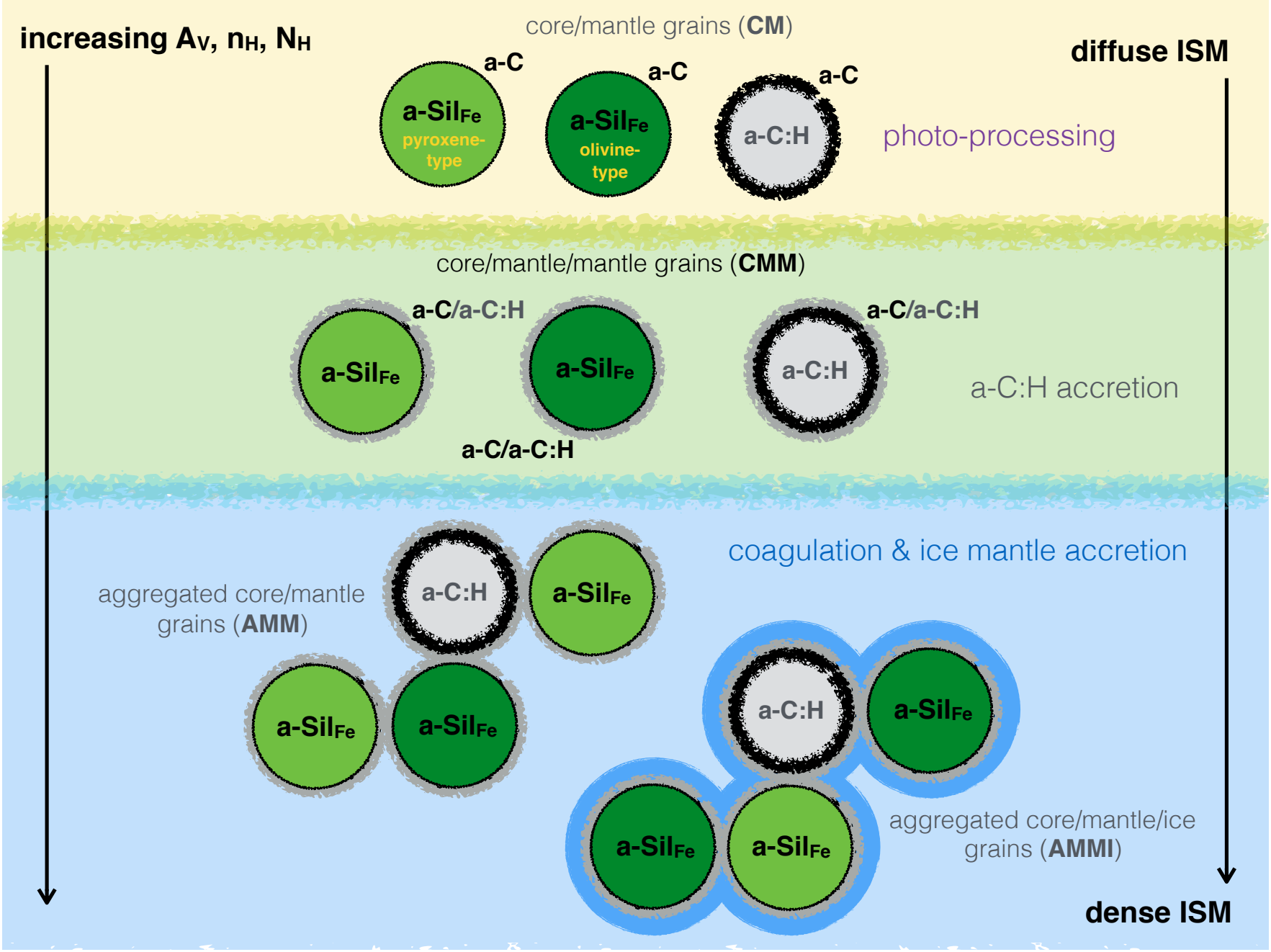

Fig. 1. Schematic view of the dust composition and stratification between diffuse ISM and dense molecular clouds. The major evolutionary processes acting on the dust in each region are indicated on the right, i.e., photo-processing, accretion and coagulation with increasing density and extinction from top to bottom.

aromatic hydrocarbons ${ }^{10}$, which could be considered as analogues of a-C(:H) nano-particles, are less stable against photodissociation than their fully aromatic equivalents. Thus, and in the absence of laboratory data and models for a-C(:H) nanoparticle excitation and photo-dissociative processing, the work of Micelotta et al. (2010b,a, 2011), which applies to fullerene and polycyclic aromatic hydrocarbons, can only be used to give an upper limit to the lifetime of a-C(:H) nano-grains with the same number of carbon atoms as fully aromatic particles.

\subsection{Nano-particle specifics}

A detailed consideration of the structure of nano-particles is explicitly treated within THEMIS (Jones 2012c), where the key considerations are particle size limits on the aromatic domain distribution and particle surface passivation through hydrogenation. This approach led us to a self-consistent explanation for the origin of the UV extinction bump in a-C nano-particles ( $a \simeq 1 \mathrm{~nm}$, Jones $2012 \mathrm{c}$ ) and also to viable routes to interstellar/circumstellar fullerene formation through UV

\footnotetext{
${ }^{10}$ N.B. Small super-hydrogenated polycyclic aromatic hydrocarbons with less than $20 \mathrm{C}$ atoms (e.g., Wolf et al. 2016) cannot be aromatic and should therefore strictly be called polycyclic aliphatic hydrocarbons.
}

photo-processing and de-hydrogenation (Bernard-Salas et al. 2012; Micelotta et al. 2012).

\subsection{Comparison with observations}

The THEMIS dust model, including the effects of amorphous pyroxene-type silicates and $\mathrm{Fe} / \mathrm{FeS}$ nano-inclusions (Köhler et al. 2014), was recently and rather favourably compared to Planck observations of dust in the diffuse ISM (Ysard et al. 2015; Fanciullo et al. 2015). It appears that physically-reasonable variations in the modelled grain compositions and structure (mantle thicknesses, metallic Fe inclusions, relative dust masses and size distributions) all lie within the range of the observations (Ysard et al. 2015). Further, an intermodel comparison using optical and Planck data showed that the THEMIS dust model fares well and appears to have correctly predicted the observed relationship between the dust optical extinction and its long wavelength emission properties in the diffuse ISM (Ysard et al. 2015; Fanciullo et al. 2015).

The THEMIS dust model includes the effects of dust evolution in the denser regions of the ISM (Jones et al. 2014; Köhler et al. 2015). The extended model (Köhler et al. 2015) is consistent with the dust scattering properties needed to explain observations of cloud-shine and core-shine (C-shine, Sect. 2.3 
and Jones et al. 2016; Ysard et al. 2016). Thus, the THEMIS modelling approach encompasses, and indeed requires, rather wide variations in the composition and size distribution from region to region within the ISM, which is supported by observational studies in addition to those of Ysard et al. (2015) and Fanciullo et al. (2015) mentioned above. Clearly, changes in the dust composition and mass can also be traced indirectly through elemental depletion studies and gas-to-dust ratio measurements in interstellar clouds.

While the depletions of the silicate-forming elements ( $\mathrm{Si}$, $\mathrm{Mg}$ and $\mathrm{Fe}$ ) do show rather wide variations they do follow general trends, with $\mathrm{Si}$ and $\mathrm{Mg}$ being more volatile than $\mathrm{Fe}$, while $\mathrm{Cr}, \mathrm{Ni}, \mathrm{Ti}$ and $\mathrm{Mn}$ tend to follow Fe and show lower but seemingly well-coupled variations (Jones 2000). These same general trends, for the same elements, are also evident in the more rigorous and complete study by Jenkins (2009), which shows that, as the depletion strengths vary, the logarithms of the depletion factors for different elements are linearly related to one another. What both of these studies show is that the depletions of the dustforming elements almost always follow the same trends, i.e., the silicate group $\mathrm{O}, \mathrm{Si}$ and $\mathrm{Mg}$ are coupled, as are the iron group $\mathrm{Fe}, \mathrm{Cr}, \mathrm{Ni}$, Ti and $\mathrm{Mn}$, which implies that these two groups of elements are each associated with a particular interstellar dust component. Interestingly, such a division into two groups is a natural consequence of the adopted THEMIS silicate grain structure and composition. The silicate group $(\mathrm{O}, \mathrm{Si}$ and $\mathrm{Mg}$ ) represent the Mg-rich olivine- and pyroxene-type amorphous silicate matrix in the large grains with the iron group $(\mathrm{Fe}, \mathrm{Cr}, \mathrm{Ni}$, $\mathrm{Ti}$ and $\mathrm{Mn}$ ) being present as Fe-rich nano-inclusions within the amorphous silicate matrix. This segregation naturally explains why the iron group elements, embedded as nano-inclusions and protected by the surrounding silicate matrix, show weaker depletion variations with respect to the surrounding silicate group elements. This dust composition and structure is entirely consistent with the most recent experimental, observational and direct interstellar silicate dust analysis indicators (see Sect. 2.1.1).

Significant cloud-to-cloud and within-cloud gas-to-dust variations are apparent in the low density ISM (low $n_{\mathrm{H}}$ ) for the same line of sight column densities $\left(N_{\mathrm{H}}\right.$, Reach et al. 2015). This work also shows a decreasing gas-to-dust ratio with decreasing dust temperature, i.e., there is an apparent dust excess in colder regions. As Reach et al. (2015) state “... grain properties may change within the clouds: they become more emissive when they are colder, while not utilizing heavy elements that already have their cosmic abundance fully locked into grains". As they noted the dust emissivity needs to be higher by about a factor of three, which is consistent with the results of Stepnik et al. (2003), Köhler et al. (2012) and, more particularly, with those of Köhler et al. (2015) for the onset of dust aggregation, i.e. the $\mathrm{CM} \rightarrow \mathrm{CMM} \rightarrow$ AMM dust evolution of the THEMIS model in the transition to denser cloud regions. The onset of these changes would seem to occur before ice mantles appear (e.g., Parvathi et al. 2012; Jones et al. 2014) and therefore indicates that accretion onto dust and dust aggregation seemingly begins before ice mantle formation (e.g., Ysard et al. 2016).

Given that the dust evolution observed by Reach et al. (2015) cannot be driven by silicate-forming elemental depletion variations, because these elements (e.g., $\mathrm{Si}, \mathrm{Mg}$ and $\mathrm{Fe}$ ) are maximally-depleted, this leaves the vestige carbon in the gas phase as the most likely accreting and dust emissivity-enhancing agent (e.g., Jones et al. 2013, 2014, 2016; Ysard et al. 2015, 2016). That significant dust variations are related to variations in the carbon depletion in the ISM are supported by observational studies (Parvathi et al. 2012; Mishra \& Li 2015).
With their measurements of interstellar carbon abundances, in both gas and dust, in environments with average hydrogen densities, $\left\langle n_{\mathrm{H}}\right\rangle$, ranging over three orders of magnitude, Parvathi et al. (2012) conclude that carbonaceous grains must be processed in the neutral ISM and that the strength of the UV bump depends neither on the carbon abundance in dust nor on the grain-size distribution. Their work supports the suggestion that big grains form in dense clouds and that they are then progressively fragmented into smaller grains in lower density regions, which is consistent with the gradual decrease in the FUV extinction in the transition to the lower density diffuse ISM. In general agreement with the results of Parvathi et al. (2012), Mishra \& Li (2015) find that carbon depletion into dust tends to correlate with $1 / R_{\mathrm{V}}$, indicating that the FUV extinction is most likely due to small carbon grains. However, in their study they find that the strength of the UV bump does tend to correlate with the depletion of carbon into dust, in contrast to the results of Parvathi et al. (2012).

Recently several aspects of the fundamental nature of interstellar dust were re-examined within the framework of the THEMIS dust model, these include: the role of carbonaceous nano-particle surface chemistry and $\mathrm{OH}$ formation in the tenuous ISM (Jones 2016a), the likely structure, form and origin of the diffuse interstellar band carriers (Jones 2016b) and a comprehensive re-exploration of the composition and evolution of core/mantle particles from the ISM to comets (Jones 2016c). The implications arising from this wide-ranging re-appraisal of the nature of cosmic dust challenge some of our long-held views, i.e., that it is chemically passive and essentially the same everywhere, but perhaps also open up new experimental and observational avenues that will lead to a deeper elucidation of its properties.

\subsection{Future developments}

In our future development of THEMIS we will include appropriate, new laboratory-measured interstellar dust analogue material optical constants as and when they become available. This will be particularly important for wavelength regions where the current model data are not sufficiently constrained by such laboratory data, i.e., for $\lambda \gtrsim 60 \mu \mathrm{m}$.

In our future extensions to and applications of THEMIS we will focus on some of the key and interesting effects that nanophysics introduces to studies of interstellar nano-particles, including: photo-electron emission and gas heating, grain charge effects and the contribution of the rotational emission from a-C grains to the dust emission spectrum.

In the near future we will calculate the polarising properties of all the THEMIS grain structures and use these to predict the interstellar polarisation by such grains.

We also plan to re-visit aspects of our studies that have to date received only preliminary analysis, such as: interactions with gas phase species (Jones et al. 2014; Jones \& Habart 2015; Jones 2016a,c), "volatile" silicon in PDRs, sulphur and nitrogen depletions in the diffuse ISM (Jones 2013, 2016a,b; Köhler et al. 2014), the origin of blue and red photoluminescence and the origin of the diffuse interstellar bands (Jones 2013, 2014, 2016b).

\section{The THEMIS model: access and links to the data}

Further details, developments and updates of the THEMIS model and modelling approach can be found on the THEMIS 
website $^{11}$, which will be updated as new data become available The THEMIS model was built and developed using the interstellar dust tool DustEM ${ }^{12}$, which calculates the dust extinction, emission, etc., when used with the appropriate input data files available via the THEMIS website. Currently the THEMIS website provides all of the input DustEM Q, C and G data files for the standard CM diffuse ISM dust model (Jones et al. 2013; Köhler et al. 2014), which are also available in the current release of DustEM. These will be complemented with other model data (CMM, AMM, AMMI, ... ) as and when these data are in a fully tested and calibrated format ready for public release. Some of the more recent work on the THEMIS model has been developed within the framework of the EU FP7-funded project DustPedi $^{13}$ and THEMIS is currently the reference dust model within the DustPedia collaboration (Davies et al. 2016).

A number of papers have already used and made reference to the THEMIS model to explore the nature and evolution of interstellar dust in the Milky Way (Jones \& Habart 2015; Jones et al. 2016; Ysard et al. 2016; Bianchi et al. 2017; De Looze et al. 2017) and within other galaxies (Bocchio et al. 2016; Viaene et al. 2016; Mosenkov et al. 2016; Clark et al. 2016; Davies et al. 2016; Chastenet et al. 2017).

\section{Summary}

THEMIS is built around a core diffuse ISM dust model and considers the evolution of this dust in response to the physical conditions in the ambient medium. The THEMIS modelling approach is global in the sense that the observable dust properties are considered as a whole, from extreme UV to $\mathrm{cm}$ wavelengths, including self-consistent spectroscopic properties, and also in the sense that it can be applied to a wide variety of interstellar environments. The modelling approach outlined here appears to provide a new view of interstellar dust and a useful framework within which many aspects of dust and its evolution in the ISM can be explored and tested.

Recent observations have shown that the nature of interstellar dust is inherently and significantly much more complex than had previously been considered. New physically-realistic dust modelling approaches, such as that adopted in the THEMIS modelling framework, need to be stoutly anchored to the laboratorymeasured properties of cosmic dust analogues, a methodology that leaves little room for arbitrary observation-fitting adjustments. The global laboratory data-constrained approach appears to be inherently more successful than models that empiricallyadjust dust properties to fit the observations. While empiricallybased dust models can be finely tuned to the observations and thereby advance astrophysical understanding, in achieving this they tend to sacrifice some physical understanding.

Acknowledgements. The authors would like to thank their numerous colleagues for the many hours of fruitful discussions on the complex subject of interstellar dust. In particular we wish to thank Alain Abergel for a careful reading of the manuscript. This research was, in part, made possible through the financial support of the Agence National de la Recherche (ANR) through the programmes Cold Dust (ANR-07-BLAN-0364- 01) and CIMMES (ANR-11BS56-0029) and, currently, through the EU FP7 funded project DustPedia (grant No. 606847)

\section{References}

Aannestad, P. A., \& Greenberg, J. M. 1983, ApJ, 272, 551

Adel, M. E., Amir, O., Kalish, R., \& Feldman, L. C. 1989, J. Appl. Phys., 66, 3248

\footnotetext{
11 http://www.ias.u-psud.fr/themis/

12 http://www.ias.u-psud.fr/dustem/

13 DustPedia.com
}

Alata, I., Cruz-Diaz, G. A., Muñoz Caro, G. M., \& Dartois, E. 2014, A\&A, 569, A119

Angus, J. C., \& Hayman, C. C. 1988, Science, 241, 913

Angus, J. C., \& Jansen, F. 1988, Journal of Vacuum Science Technology, 6, 1778 Bernard-Salas, J., Cami, J., Peeters, E., et al. 2012, ApJ, 757, 41

Bianchi, S., Giovanardi, C., Smith, M. W. L., et al. 2017, A\&A, 597, A130 Bocchio, M., Micelotta, E. R., Gautier, A.-L., \& Jones, A. P. 2012, A\&A, 545, A124

Bocchio, M., Jones, A. P., Verstraete, L., et al. 2013, A\&A, 556, A6

Bocchio, M., Jones, A. P., \& Slavin, J. D. 2014, A\&A, 570, A32

Bocchio, M., Bianchi, S., Hunt, L. K., \& Schneider, R. 2016, A\&A, 586, A8

Bohren, C. F., \& Huffman, D. R. 1998, Absorption and Scattering of Light by

Small Particles (New York, Chichester, Brisbane, Toronto, Singapore: Wiley and Sons)

Bradley, J. P. 1994, Science, 265, 925

Bron, E., Le Bourlot, J., \& Le Petit, F. 2014, A\&A, 569, A100

Cecchi-Pestellini, C., Cacciola, A., Iatì, M. A., et al. 2010, MNRAS, 408, 535

Cecchi-Pestellini, C., Casu, S., Mulas, G., \& Zonca, A. 2014a, ApJ, 785, 41

Cecchi-Pestellini, C., Viti, S., \& Williams, D. A. 2014b, ApJ, 788, 100

Chastenet, J., Bot, C., Gordon, K. D., et al. 2017, A\&A, 601, A55

Chiar, J. E., Tielens, A. G. G. M., Whittet, D. C. B., et al. 2000, ApJ, 537, 749

Clark, C. J. R., Schofield, S. P., Gomez, H. L., \& Davies, J. I. 2016, MNRAS, 459, 1646

Compiègne, M., Verstraete, L., Jones, A., et al. 2011, A\&A, 525, A103

Costantini, E., Freyberg, M. J., \& Predehl, P. 2005, A\&A, 444, 187

Costantini, E., Pinto, C., Kaastra, J. S., et al. 2012, A\&A, 539, A32

Crinklaw, G., Federman, S. R., \& Joseph, C. L. 1994, ApJ, 424, 748

Dalle Ore, C. M., Fulchignoni, M., Cruikshank, D. P., et al. 2011, A\&A, 533, A98

Dartois, E., \& Muñoz-Caro, G. M. 2007, A\&A, 476, 1235

Dartois, E., Marco, O., Muñoz-Caro, G. M., et al. 2004a, A\&A, 423, 549

Dartois, E., Muñoz Caro, G. M., Deboffle, D., \& d'Hendecourt, L. 2004b, A\&A, 423, L33

Dartois, E., Muñoz Caro, G. M., Deboffle, D., Montagnac, G., \& D’Hendecourt, L. 2005, A\&A, 432, 895

Davies, J. I., Baes, M., Bianchi, S., et al. 2016, PASP, 129, 974

Davoisne, C., Djouadi, Z., Leroux, H., et al. 2006, A\&A, 448, L1

De Looze, I., Barlow, M. J., Swinyard, B. M., et al. 2017, MNRAS, 465, 3309

Demyk, K., Carrez, P., Leroux, H., et al. 2001, A\&A, 368, L38

Demyk, K., d'Hendecourt, L., Leroux, H., Jones, A. P., \& Borg, J. 2004, A\&A, 420, 233

Desert, F.-X., Boulanger, F., \& Puget, J. L. 1990, A\&A, 237, 215

Döhler, G. H., Dandaloff, R., \& Bilz, H. 1980, J. Noncryst. Solids, 42, 87

Draine, B. T., \& Flatau, P. J. 2000, Astrophysics Source Code Library [record ascl: 0008.001$]$

Draine, B. T., \& Lee, H. M. 1984, ApJ, 285, 89

Draine, B. T., \& Li, A. 2001, ApJ, 551, 807

Draine, B. T., \& Li, A. 2007, ApJ, 657, 810

Duley, W. W. 1984, ApJ, 287, 694

Duley, W. W. 1987, MNRAS, 229, 203

Duley, W. W. 2001, ApJ, 553, 575

Duley, W. W., Jones, A. P., \& Williams, D. A. 1989, MNRAS, 236, 709

Duley, W. W., Seahra, S., \& Williams, D. A. 1997, ApJ, 482, 866

Dwek, E., Arendt, R. G., Fixsen, D. J., et al. 1997, ApJ, 475, 565

Fanciullo, L., Guillet, V., Aniano, G., et al. 2015, A\&A, 580, A136

Ferrari, A. C., \& Robertson, J. 2000, Phys. Rev. B, 61, 14095

Ferrari, A. C., \& Robertson, J. 2004, Phil. Trans. R. Soc. Lond. A, 362, 2477

Gadallah, K. A. K., Mutschke, H., \& Jäger, C. 2011, A\&A, 528, A56

Galliano, F., Hony, S., Bernard, J.-P., et al. 2011, A\&A, 536, A88

Garnett, J. C. M. 1904, Phil. Trans. R. Soc. A: Math., 203, 385

Gavilan, L., Alata, I., Le, K. C., et al. 2016, A\&A, 586, A106

Godard, M., \& Dartois, E. 2010, A\&A, 519, A39

Godard, M., Féraud, G., Chabot, M., et al. 2011, A\&A, 529, A146

Goto, M., Gaessler, W., Hayano, Y., et al. 2003, ApJ, 589, 419

Goto, M., Kwok, S., Takami, H., et al. 2007, ApJ, 662, 389

Greenberg, J. M. 1986, Ap\&SS, 128, 17

Greenberg, J. M., \& Chlewicki, G. 1983, ApJ, 272, 563

Hecht, J. H. 1986, ApJ, 305, 817

Hockey, T., Trimble, V., Williams, T. R., et al. 2014, Biographical Encyclopedia of Astronomers (Berlin: Springer)

Hollenbach, D., \& Salpeter, E. E. 1971, ApJ, 163, 155

Hoyle, F., \& Wickramasinghe, N. C. 1962, MNRAS, 124, 417

Hoyle, F., \& Wickramasinghe, N. C. 1969, Nature, 223, 459

Iatì, M. A., Saija, R., Borghese, F., et al. 2008, MNRAS, 384, 591

Jenkins, E. B. 2009, ApJ, 700, 1299

Jones, A. P. 1988, MNRAS, 234, 209

Jones, A. P. 1990, MNRAS, 247, 305

Jones, A. P. 2000, J. Geophys. Res., 105, 10257 
Jones, A. P. 2012a, A\&A, 540, A1

Jones, A. P. 2012b, A\&A, 540, A2; Corrigendum: 545, C2

Jones, A. P. 2012c, A\&A, 542, A98; Corrigendum: 545, C3

Jones, A. P. 2013, A\&A, 555, A39

Jones, A. P. 2014, Planet. Space Sci., 100, 26

Jones, A. P. 2015, IAU General Assembly, 22, 2256693

Jones, A. P. 2016a, R. Soc. Open Sci., 3, 160221

Jones, A. P. 2016b, R. Soc. Open Sci., 3, 160223

Jones, A. P. 2016c, R. Soc. Open Sci., 3, 160224

Jones, A. P., \& Habart, E. 2015, A\&A, 581, A92

Jones, A. P., Williams, D. A., \& Duley, W. W. 1987, MNRAS, 229, 213

Jones, A. P., Duley, W. W., \& Williams, D. A. 1990, QJRAS, 31, 567

Jones, A. P., Fanciullo, L., Köhler, M., et al. 2013, A\&A, 558, A62

Jones, A. P., Ysard, N., Köhler, M., et al. 2014, RSC Faraday Discuss., 168, 313

Jones, A. P., Köhler, M., Ysard, N., et al. 2016, A\&A, 588, A43

Jura, M. 1980, ApJ, 235, 63

Kassavetis, S., Patsalas, P., Logothetidis, S., Robertson, J., \& Kennou, S. 2007, Diamond \& Related Materials, 16, 1813

Kimoto, S., Dick, W. D., Syedain, Z., Pui, D. Y. H., \& Roberts, D. L. 2014, in International Aerosol Conference, Korea, PP08-016

Köhler, M., Guillet, V., \& Jones, A. 2011, A\&A, 528, A96

Köhler, M., Stepnik, B., Jones, A. P., et al. 2012, A\&A, 548, A61

Köhler, M., Jones, A., \& Ysard, N. 2014, A\&A, 565, L9

Köhler, M., Ysard, N., \& Jones, A. P. 2015, A\&A, 579, A15

Le Page, V., Snow, T. P., \& Bierbaum, V. M. 2009, ApJ, 704, 274

Lefevre, J. 1974, A\&A, 37, 17

Li, A., \& Draine, B. T. 2001, ApJ, 554, 778

Li, A., \& Draine, B. T. 2002, ApJ, 576, 762

Li, A., \& Greenberg, J. M. 1997, A\&A, 323, 566

Llamas-Jansa, I., Jäger, C., Mutschke, H., \& Henning, T. 2007, Carbon, 45, 1542

Marée, C. H. M., Vredenberg, A. M., \& Habraken, F. H. P. M. 1996, Materials Chemistry and Physics, 46, 198

Mathis, J. S., \& Whiffen, G. 1989, ApJ, 341, 808

Mathis, J. S., Rumpl, W., \& Nordsieck, K. H. 1977, ApJ, 217, 425

Mennella, V. 2008, ApJ, 682, L101

Mennella, V. 2010, ApJ, 718, 867

Mennella, V., Colangeli, L., Bussoletti, E., et al. 1995, ApJS, 100, 149

Mennella, V., Colangeli, L., Palumbo, P., et al. 1996, ApJ, 464, L191

Mennella, V., Baratta, G. A., Esposito, A., Ferini, G., \& Pendleton, Y. J. 2003, ApJ, 587, 727

Micelotta, E. R., Jones, A. P., \& Tielens, A. G. G. M. 2010a, A\&A, 510, A37

Micelotta, E. R., Jones, A. P., \& Tielens, A. G. G. M. 2010b, A\&A, 510, A36

Micelotta, E. R., Jones, A. P., \& Tielens, A. G. G. M. 2011, A\&A, 526, A52

Micelotta, E. R., Jones, A. P., Cami, J., et al. 2012, ApJ, 761, 35

Mie, G. 1908, Annalen der Physik, 330, 377

Mishra, A., \& Li, A. 2015, ApJ, 809, 120

Mosenkov, A. V., Allaert, F., Baes, M., et al. 2016, A\&A, 592, A71

Muñoz Caro, G. M., Matrajt, G., Dartois, E., et al. 2006, A\&A, 459, 147

Oort, J. H., \& van de Hulst, H. C. 1946, Bull. Ast. Inst. Netherlands, 10, 187

Ordal, M. A., Bell, R. J., Alexander, Jr., R. W., Long, L. L., \& Querry, M. R. 1985, Appl. Opt., 24, 4493

Ordal, M. A., Bell, R. J., Alexander, Jr., R. W., Newquist, L. A., \& Querry, M. R. 1988, Appl. Opt., 27, 1203

Ordal, M. A., Long, L. L., Bell, R. J., et al. 1983, Appl. Opt., 22, 1099

Ossenkopf, V. 1993, A\&A, 280, 617

Parvathi, V. S., Sofia, U. J., Murthy, J., \& Babu, B. R. S. 2012, ApJ, 760, 36
Pendleton, Y. J., \& Allamandola, L. J. 2002, ApJS, 138, 75

Phillips, J. C. 1979, Phys. Rev. Lett., 42, 1151

Pino, T., Dartois, E., Cao, A., et al. 2008, A\&A, 490, 665

Pollack, J. B., Hollenbach, D., Beckwith, S., et al. 1994, ApJ, 421, 615

Rauls, E., \& Hornekær, L. 2008, ApJ, 679, 531

Reach, W. T., Heiles, C., \& Bernard, J.-P. 2015, ApJ, 811, 118

Robertson, J. 1986, Advances in Physics, 35, 317

Robertson, J. 1988, Phil. Mag. Lett., 57, 143

Robertson, J. 1991, Prog. Solid State Chem., 21, 199

Robertson, J. 2001, Physica Status Solidi Applied Research, 186, 1521

Robertson, J. 2002, Mat. Sci. Eng., 37, 129

Robertson, J., \& O’Reilly, E. P. 1987, Phys. Rev. B, 35, 2946

Rouleau, F., \& Martin, P. G. 1991, ApJ, 377, 526

Routly, P. M., \& Spitzer, Jr., L. 1952, ApJ, 115, 227

Savage, B. D., \& Sembach, K. R. 1996, ARA\&A, 34, 279

Schalén, C. 1929, Astron. Nachr., 236, 249

Schalén, C. 1931, Publ. Am. Astron. Soc., 6, 376

Schalén, C. 1934, Meddel. Astron. Obs. Upsala, 58

Schoenberg, E., \& Jung, B. 1934, Astron. Nachr., 253, 261

Scott, A., \& Duley, W. W. 1996, ApJS, 105, 401

Serra Díaz-Cano, L., \& Jones, A. P. 2008, A\&A, 492, 127

Siebenmorgen, R., \& Kruegel, E. 1992, A\&A, 259, 614

Siebenmorgen, R., Voshchinnikov, N. V., \& Bagnulo, S. 2014, A\&A, 561, A82

Sloan, G. C., Jura, M., Duley, W. W., et al. 2007, ApJ, 664, 1144

Smith, F. W. 1984, J. Appl. Phys., 55, 764

Sorrell, W. H. 1990, MNRAS, 243, 570

Sorrell, W. H. 1991, MNRAS, 248, 439

Stecher, T. P. 1965, ApJ, 142, 1683

Stecher, T. P. 1969, ApJ, 157, L125

Stecher, T. P., \& Donn, B. 1965, ApJ, 142, 1681

Stepnik, B., Abergel, A., Bernard, J.-P., et al. 2003, A\&A, 398, 551

Tamor, M. A., \& Wu, C. H. 1990, J. Appl. Phys., 67, 1007

Thorpe, M. 1983, Journal of Non Crystalline Solids, 57, 355

Trumpler, R. J. 1930, PASP, 42, 214

van de Hulst, H. C. 1943, Ned. Tijdschr. V. Natuur, 10, 251

Viaene, S., Baes, M., Bendo, G., et al. 2016, A\&A, 586, A13

Warren, S. G. 1984, Applied Optics, 23, 1206

Welty, D. E., Jenkins, E. B., Raymond, J. C., Mallouris, C., \& York, D. G. 2002, ApJ, 579, 304

Westphal, A. J., Stroud, R. M., Bechtel, H. A., et al. 2014, Science, 345, 786

Whittet, D. C. B., Martin, P. G., Hough, J. H., et al. 1992, ApJ, 386, 562

Whittet, D. C. B., Gerakines, P. A., Hough, J. H., \& Shenoy, S. S. 2001, ApJ, 547,872

Wickramasinghe, N. C. 1963, MNRAS, 126, 99

Wickramasinghe, N. C. 1970, in Ultraviolet Stellar Spectra and Related GroundBased Observations, eds. R. Muller, L. Houziaux, \& H. E. Butler, IAU Symp., 36,42

Wolf, M., Kiefer, H. V., Langeland, J., et al. 2016, ApJ, 832, 24

Xiang, J., Lee, J. C., Nowak, M. A., \& Wilms, J. 2011, ApJ, 738, 78

Ysard, N., Köhler, M., Jones, A., et al. 2015, A\&A, 577, A110

Ysard, N., Köhler, M., Jones, A., et al. 2016, A\&A, 588, A44

Zonca, A., Cecchi-Pestellini, C., Mulas, G., \& Malloci, G. 2011, MNRAS, 410, 1932

Zubko, V., Dwek, E., \& Arendt, R. G. 2004, ApJS, 152, 211

Zubko, V. G., Mennella, V., Colangeli, L., \& Bussoletti, E. 1996, MNRAS, 282, 1321 